\title{
Leakage flux modelling of multi-winding transformer using permeance magnetic cir- cuit
}

M. Luo, D. Dujic, and J. Allmeling

This material is posted here with permission of the IEEE. Such permission of the IEEE does not in any way imply IEEE endorsement of any of EPFL's products or services. Internal or personal use of this material is permitted. However, permission to reprint / republish this material for advertising or promotional purposes or for creating new collective works for resale or redistribution must be obtained from the IEEE by writing to pubs-permissions@ieee. org. By choosing to view this document, you agree to all provisions of the copyright laws protecting it. 


\section{Leakage Flux Modelling of Multi-Winding Transformer using Permeance Magnetic Circuit}

\author{
Min Luo and Drazen Dujic \\ Power Electronics Laboratory \\ École Polytechnique Fédérale de Lausanne (EPFL) \\ Lausanne CH-1015, Switzerland \\ min.luo@epfl.ch,drazen.dujic@epfl.ch
}

\author{
Jost Allmeling \\ Plexim GmbH \\ Zürich CH-8005, Switzerland \\ allmeling@plexim.com
}

\begin{abstract}
The size and position difference of the windings determine the leakage flux path and give rise to unbalance of the short-circuit impedances, which strongly affects the transient behaviour of the transformer. This work proposes a new approach of modelling with magnetic equivalent circuit, making use of the transformer geometry and permeance magnetic equivalent circuit, which is suitable for system-level simulation in terms of complexity. Equivalent model requires limited number of parameters and for verification purposes, FEM simulations as well as measurement on the experimental prototype have been performed.
\end{abstract}

\section{INTRODUCTION}

Multi-winding transformers are adopted in power electronics system for transferring power between multiple terminals. Different size and position make the leakage coupling between some windings stronger than the others, which give rise to unbalanced short-circuit impedance values or non-symmetrical conditions during operation.

Finite element method (FEM) is able to accurately model the leakage field, however it is not suitable for transient simulation of large power electronics system due to the high computational effort. For this purpose electrical equivalent circuit with coupled inductors is commonly used. This type of model requires hardware test to identify the magnetisation inductance $L_{m}$ and the leakage inductance $L_{\sigma}$. If more than two windings are present, the short-circuit test needs to be enumerated among all the winding pairs, which can be time consuming or in some cases impractical.

Magnetic circuit is closely related to geometry and material parameters, which is intuitive and usually easier to obtain compared to the hardware test results. Existing publications based on this methodology have modelled the iron core in quite deep details level like [1], [2] and [3], in which however the leakage path was rather roughly represented. Authors of [4] included the leakage flux path into the magnetic circuit, and proposed a way to quantitatively calculate the parameters. But this approach was only validated by a FEM-simulated single-winding inductor with simple topology. In the work of [5] authors aimed at more complicated cases, where the leakage flux path was also integrated into the magnetic circuit and the result was compared with FEM simulation of multiwinding transformers. The work of [6] further on verified [5]'s approach via experimental test. Typical winding arrangements like multilayer- (Fig. 1a) and multidisk- (Fig. 1b) structures

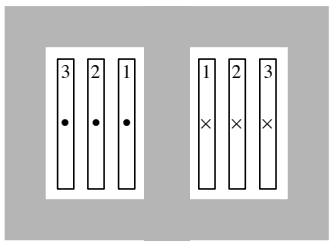

(a) Multilayer

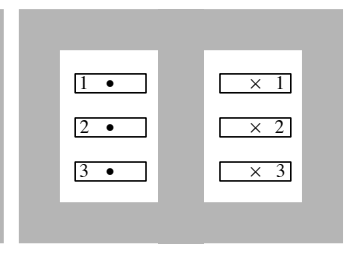

(b) Multidisk

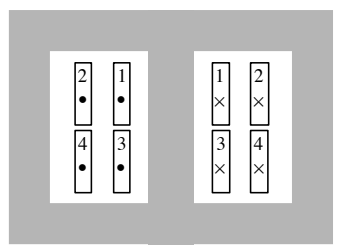

(c) Mixed

Fig. 1: Typical winding structures considered in the existing literature

have been investigated, as well as a mixed case (Fig. 1c). But the topology shown in Fig. 2 has not been discussed, where the height of the inner layer winding is much larger than the others. This topology is typical for the phase-shift transformers used in multi-pulse rectifier systems, which interface power grid to medium voltage power electronics system like the cascaded H-bridge converter [7].

Moreover, in both publications the magnetic circuit was finally transformed back to electrical equivalent with coupled inductors before parametrisation. The model in [5] required to obtain the short-circuit impedances of all the winding pairs via experimental measurement, in order to derive the coupled inductance matrix of the electrical equivalent circuit. Making use of the approach from [8], authors of [6] managed to calculate single short-circuit impedance based on geometry information. However the characteristic of the core was not modelled in a straight forward way. Instead of obtained from the geometry and material characteristic or open circuit test, the information about main flux path in the core was indirectly derived from the short-circuit impedance, where the core characteristic only has slight affect. This may result in significant error when simulating light load conditions when the main flux path inside the core dominates the transient behaviour, especially when 


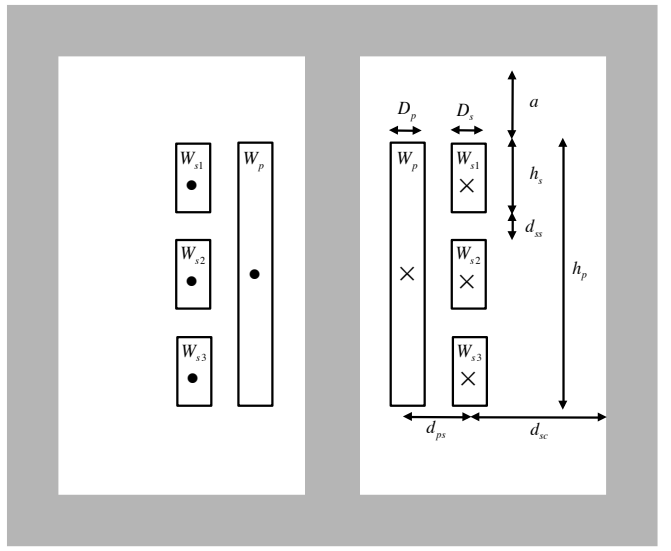

Fig. 2: Winding structure considered in this work, with the height of primary winding significantly larger than the secondary ones

taking into account the nonlinearity of the core material.

This paper deals with direct magnetic circuit modelling of the special transformer topology in Fig. 2 with a large number of winding, which is directly integrated into electric circuit simulation without being transformed back to electrical equivalent. The permeance-capacitor representation proposed by [9] and implemented by [10] is adopted, considering its high efficiency for circuit simulation. The core- and the leakage-flux path are modelled separately in the magnetic circuit, which provides the possibility to accurately represent the core's characteristic. In particular, the emphasis is placed on the modelling of various leakage flux paths, that may be encountered in these type of transformers. Especially when the windings' geometry has repetitive property, the parametrisation of the model can be simplified. For parameter identification, core geometry and material characteristic together with only a limited number of short circuit test are required. FEM simulations and a small scale prototype transformer are used to confirm validity of proposed modelling. Single-phase case is considered in this work.

\section{ANALYSIS}

Assuming that all secondary windings $W_{s n}(n=1 \ldots 3)$ of the transformer in Fig. 2 are $h_{s}$ high and $D_{s}$ thick, they are allocated along the middle core limb with the same vertical interval $d_{s s} . d_{s s}$ is usually significantly smaller than $h_{s}$ and $D_{s}$ in these types of transformers. The height of the primary winding $h_{p}$ at the inner layer is equal to the distance from the top of $W_{s 1}$ to the bottom of $W_{s 3}$. This can be interpreted into a magnetic circuit demonstrated in Fig. 3 assuming that both the air- and core-flux are flowing through the defined permeance channels. The permeances filled with yellow color represent flux path inside the core, while the others represent the leakage flux path through the air. Please note that the long primary winding $W_{p}$ has been separated into 3 sections aligned with each of the secondary windings. If all the windings are wounded close enough to the middle limb of the transformer core (as is usually the case in practice) the horizontal leakage permeances above- or below the windings can be assumed to

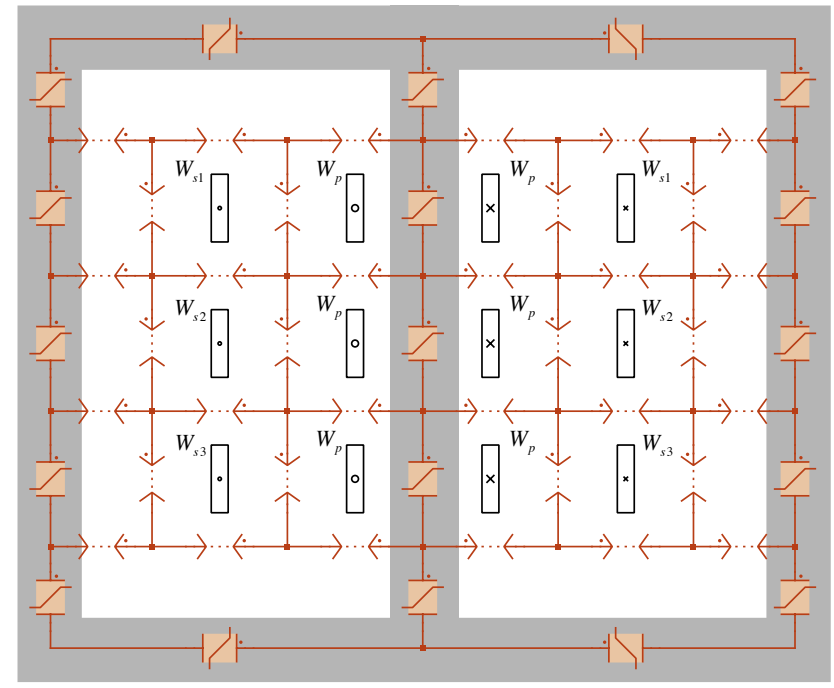

Fig. 3: Preliminary permeance circuit of the transformer, where the flux path of the core and air are illustrated

be short circuit. Moreover the physically depicted windings can be interpreted into lumped-components by means of inserting several fictitious windings $W_{s}^{\prime}$ and $W_{s}$ ", as required by the magnetic equivalent circuit. The fictitious windings have the same number of turns as the physical ones and wired in opposite directions, so that they cancel each other and do not have netto influence on the system. The permeance circuit with fictitious windings is demonstrated in Fig. 4.

Combining the physical $W_{s}$ and the fictitious $W_{s}^{\prime}$ into single winding components on the left- and right-side respectively, then placing $W_{s}$ " on the middle limb, the permeance network can be transformed into the form as shown in Fig.

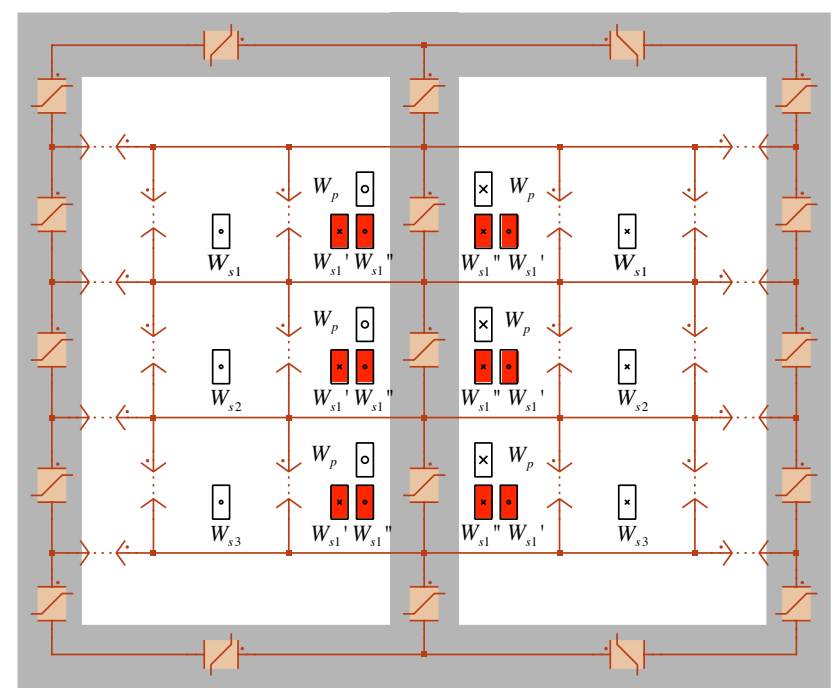

Fig. 4: Permeance circuit with horizontal permeance neglected and insertion of fictitious windings 


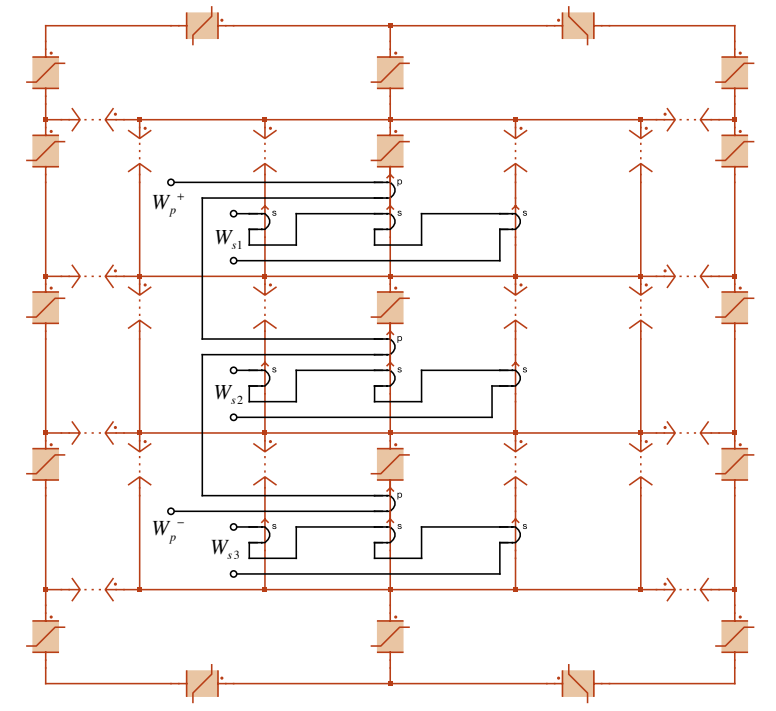

Fig. 5: Permeance circuit with lumped-winding components to interface electrical circuit

5, which is able to be directly connected to electrical circuit model. The lumped winding components are implemented using the gyrator structure proposed by [9], which serves as interface between the electrical and magnetic circuit shown in Fig. 6. On one hand, the electrical voltage measured on the winding terminal is divided by the number of turns $N$ and fed into the magnetic circuit as a flux rate source $(d \Phi / d t)$. The flux rate source will charge or discharge the magnetic permeances which behave like a capacitor. On the other hand, the "voltage" measured on the magnetic terminal (MMF) is also divided by $N$ and fed into the electrical circuit as current source.

Please note that all the primary winding sections as well as the secondary windings together with their fictitious counterparts are connected in series respectively in the electrical circuit. For this single phase transformer, the left side can be mirror to the right side due to the symmetry about the middle limb of the core, so that the model can be further simplified as shown in Fig. 7. Here the core permeances are calculated as

$$
P=\frac{\mu_{r} \mu_{0} A}{l}
$$

The equivalent cross-section area $A$ and magnetic path length $l$ can be calculated from the core geometry according to [11]. For this case, the length of $P_{\text {midn }}$ and $P_{\text {siden }}$ is equal to $h_{s}+d_{s s}$, while that of $P_{\operatorname{midT}(B)}$ and $P_{\operatorname{sideT}(B)}$ equal to $a$.

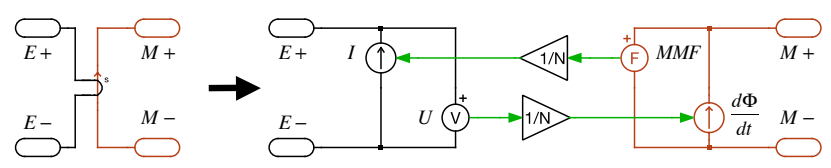

Fig. 6: Gyrator structure of the lumped-winding component

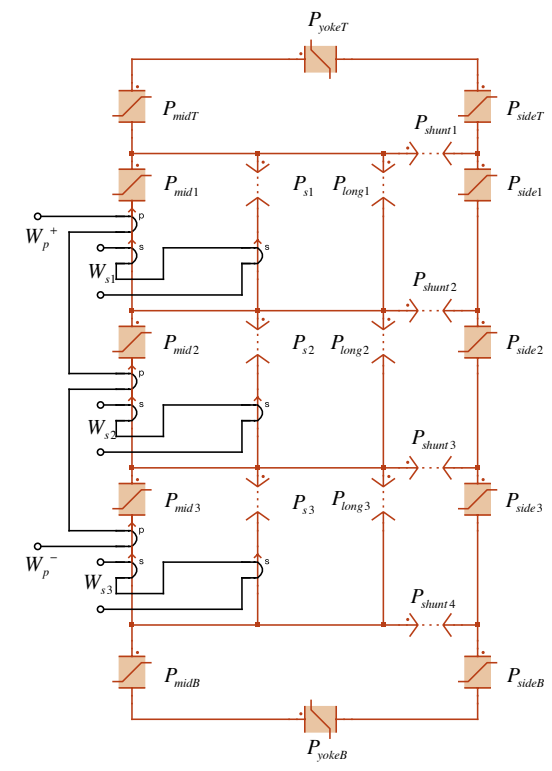

Fig. 7: Mirrored permeance network used to model multiwinding single phase transformer

The permeance $P_{y o k e T(B)}$ includes both the yoke and corner. Should nonlinearity of the core material be considered, the relative permeance $\mu_{r}$ will be a function of the magnetomotive force $F$, which is the magnetic "voltage" measure on the individual permeance. The approach proposed by [12] can also be applied to model the corner area of the core more accurately.

Up to this stage only the leakage permeances $P_{s}, P_{\text {long }}$ and $P_{\text {shunt }}$ are still unknown. Considering the fact that all the secondary windings have the same size and are located homogeneously along the middle limb, all $P_{s n}, P_{\text {longn }}$ and $P_{\text {shuntn }}$ $(n=1,2,3)$ are assumed to have the same value respectively. Since it is difficult to accurately define the geometry of the air flux path, short circuit test will be carried out to identify the three unknown parameters. In the next section, it will demonstrated that the same structure can be easily extended to the case with more than 3 secondary windings.

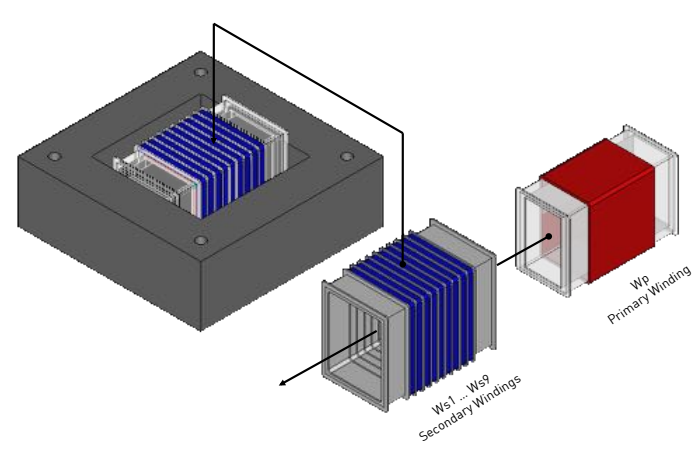

Fig. 8: Multiwinding transformer prototype 


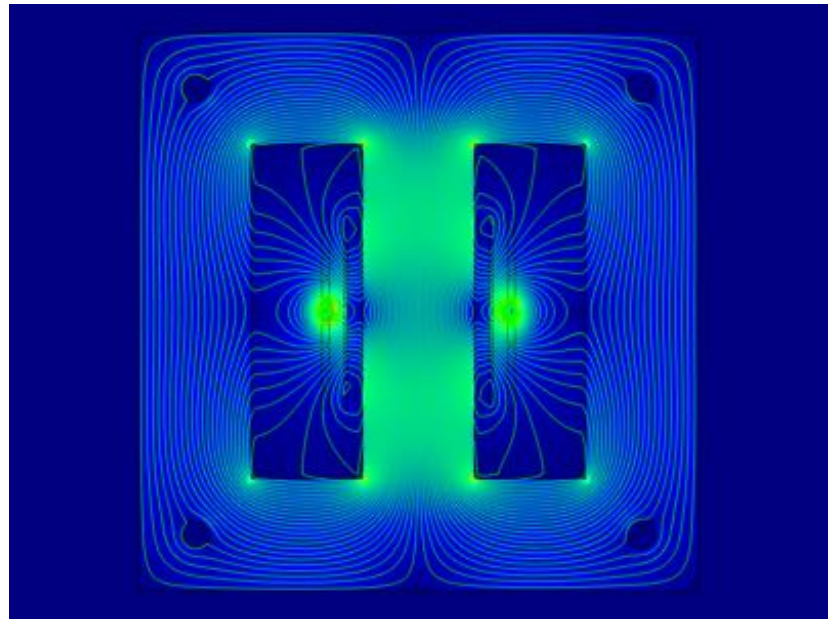

Fig. 9: Flux distribution with $W_{p}$ excited and $W_{s 5}$ shorted

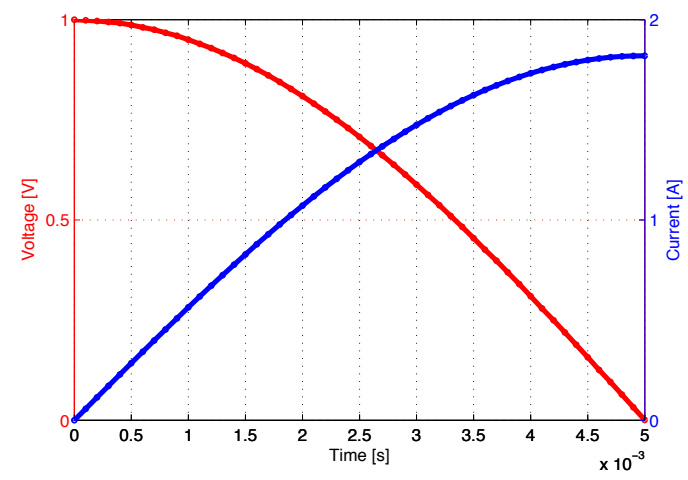

Fig. 10: $W_{p}$ current and voltage with $W_{p}$ excited, $W_{s 5}$ shorted

\section{VALIDATION}

In order to validate the fidelity of the proposed modelling's approach, a low-power, single-phase transformer prototype has been built up as depicted in Fig. 8. The prototype three-limb transformer core $(50 \mathrm{~mm}$ thick) is made of standard silicon steel laminations type "EI 150/150" of material "M330-35A". The primary winding $\left(W_{p}\right)$ has $N_{p}=193$ turns which spreads along the middle limb with $h_{p}=53 \mathrm{~mm}$ height and $1 \mathrm{~mm}$ thick. The nine secondary windings $\left(W_{s 1}-W_{s 9}\right)$ have Ns $=30$ turns each and are installed outside the primary winding, $h_{s}=$ $5 \mathrm{~mm}$ high and $2 \mathrm{~mm}$ thick, stacking vertically over eachother with $d_{s s}=1 \mathrm{~mm}$ interval in between.

A 2-dimensional FEM model has been made in COMSOL according to the real geometry, considering its accuracy in simulating leakage flux distribution. Two kinds of schemes are simulated in FEM. Firstly, sinusoidal voltage excitation with $1 \mathrm{~V}$ amplitude is applied on primary winding $W_{p}$ with single secondary winding shorted. Fig. 9 shows the flux density distribution at the current peak from FEM simulation, and Fig. 10

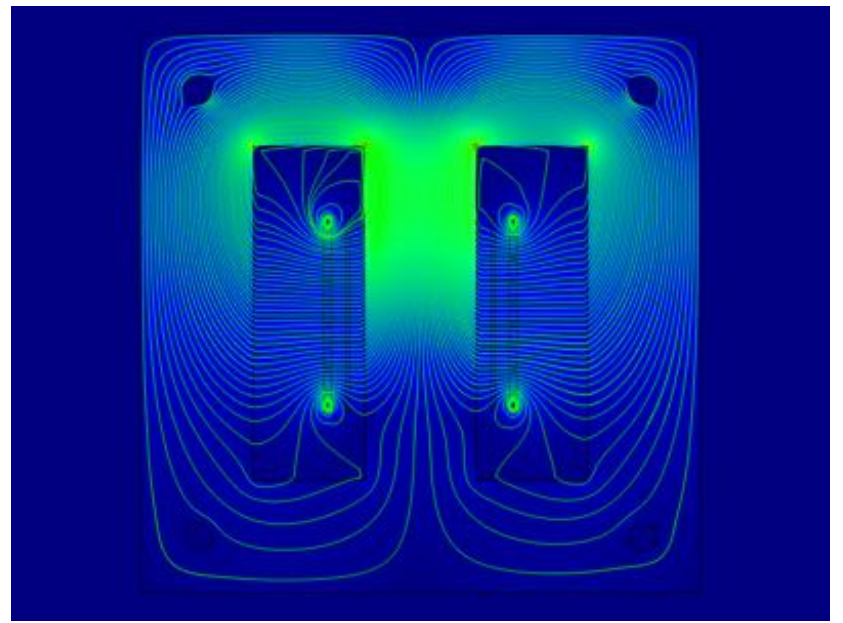

Fig. 11: Flux distribution with $W_{s 1}$ excited and $W_{s 9}$ shorted

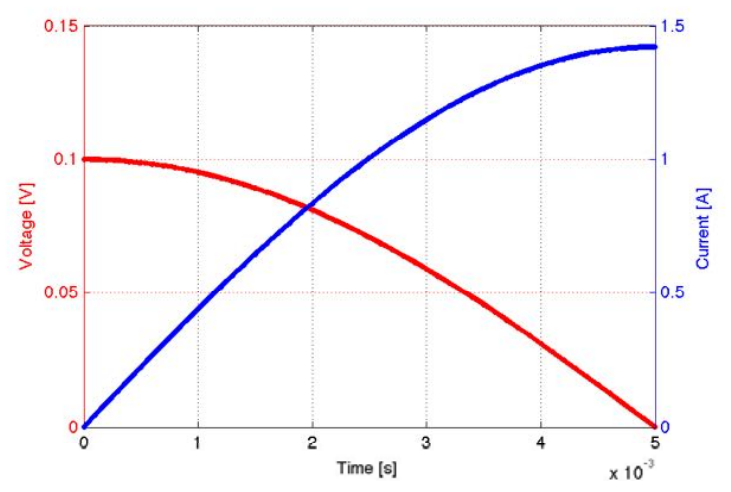

Fig. 12: $W_{p}$ current and voltage with $W_{s 1}$ excited, $W_{s 9}$ shorted

shows time-domain winding current and voltage waveform of $1 / 4 \mathrm{AC}$ period, in the scheme when the fifth secondary winding $W_{s 5}$ is shorted. The short-circuit impedance is calculated via dividing the peak value of the voltage by the current

$$
Z_{k}=\hat{V}_{W p} / \hat{I}_{W p}
$$

Resistivity of the windings and laminations is neglected in this model, so that $Z_{k}$ is purely inductive, therefore the shortcircuit inductance can be calculated directly as

$$
L_{k}=Z_{k} /(2 \pi f)
$$

Secondly, sinusoidal voltage with $0.1 \mathrm{~V}$ amplitude is applied on one secondary winding, while another secondary winding is shorted. The short impedance is calculated as well by $\hat{V}_{W s} / \hat{I}_{W s}$. Fig. 11 and Fig. 12 display the simulation result when $W_{s 1}$ is excited and $W_{s 9}$ shorted. 


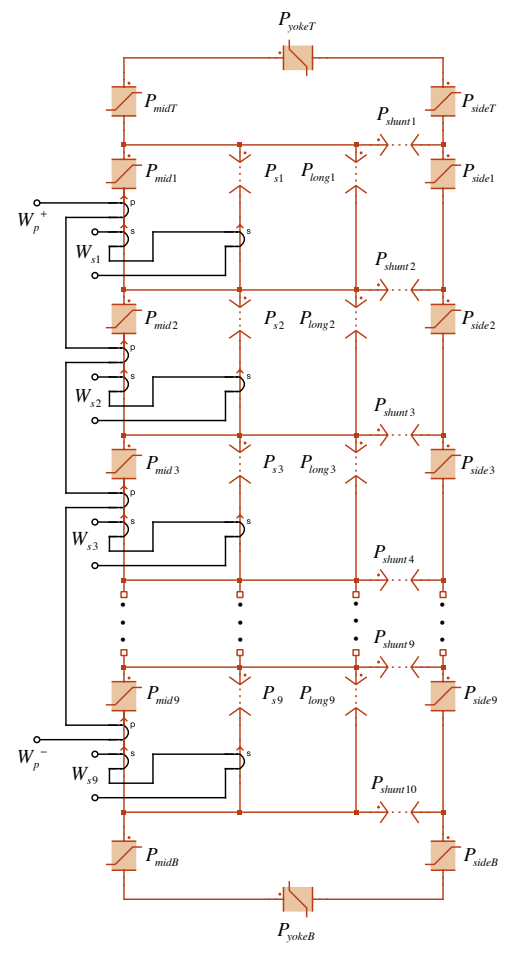

Fig. 13: Permeance circuit model of the prototype transformer

Afterwards the model based on permeance magnetic circuit is built up in the system-level simulation platform PLECS for power electronics, making use of its magnetic component library [10]. This mode is essentially an extension of the transformer example from the last section as shown in Fig. 13. As discussed in section II, the permeances representing the core $\left(P_{\text {midn }}, P_{\text {siden }}(n=1 \ldots 9), P_{\text {midT }(B)}, P_{\text {sideT(B) }}\right.$, $\left.P_{\text {yokeT(B) }}\right)$ can be directly parametrised using the geometrical and material information, whose values are listed in TABLE. I.

TABLE I: Core permeance values $[W b / A]$

\begin{tabular}{c||c||c||c||c}
\hline$P_{\text {midn }}$ & $P_{\text {midT }(B)}$ & $P_{\text {siden }}$ & $P_{\text {sideT }(B)}$ & $P_{\text {yokeT }(B)}$ \\
\hline \hline $3.13 \cdot 10^{-4}$ & $1.04 \cdot 10^{-4}$ & $6.26 \cdot 10^{-4}$ & $2.09 \cdot 10^{-4}$ & $3.02 \cdot 10^{-5}$ \\
\hline
\end{tabular}

The magnetisation characteristic (B-H curve) of the material "M330-35A" can be specified to the core permeances using atan fit to representing the saturation effect. Moreover, if the hysteresis loop is available from experimental test, Preisach model with Lorentzian distribution function proposed by [13] can be directly applied to the permeance implementation as well. Since focus of this work is on leakage flux, only the characteristic in the linear area of the $\mathrm{B}-\mathrm{H}$ curve has been assigned to the permeances, while the effect of the nonlinearity like saturation and hysteresis will be discussed in the future work.

Apart from the core permeances, the 3 values of the leakage permeances $\left(P_{s}, P_{\text {long }}, P_{\text {shunt }}\right)$ is obtained via fitting of test results (FEM or experimental). The short-circuit impedance values of 4 short-circuit test schemes are used for the parameter fitting, which essentially compose optimisation problem to minimise a quadratic objective function, described as

$$
f\left(P_{s}, P_{\text {long }}, P_{\text {shunt }}\right)=\sum_{i=1}^{4}\left(\frac{L_{k i, T e s t}-L_{k i, \text { Sim }}}{L_{k i, T e s t}}\right)^{2}
$$

- $\quad L_{k 1, T e s t}$ measured with $W_{p}$ excited and $W_{s 1}$ shorted

- $\quad L_{k 2, T e s t}$ measured with $W_{p}$ excited and $W_{s 5}$ shorted

- $\quad L_{k 3, T e s t}$ measured with $W_{s 1}$ excited and $W_{s 2}$ shorted

- $\quad L_{k 4, T e s t}$ measured with $W_{s 1}$ excited and $W_{s 9}$ shorted

Commonly used gradient based- or evolutionary algorithms can be applied to solve this problem. Initial values are configured in order to make the algorithm converge fast, which in this case can be calculated as

$$
\begin{gathered}
P_{s 0}=2 \cdot \frac{\mu_{r} \mu_{0} D_{c} d_{p s}}{h_{s}+d_{s s}} \\
P_{\text {long } 0}=0 \\
P_{\text {shunt } 0}=2 \cdot \frac{\mu_{r} \mu_{0} D_{c}\left(h_{s}+d_{s s}\right)}{d_{s c}}
\end{gathered}
$$

$D_{c}=50 \mathrm{~mm}$ is the thickness of the core, while other geometrical parameters present in the equation above have been defined in Fig. 2. The "fminsearch" function provided by MATLAB (referred as unconstrained nonlinear optimisation) is adopted for this case, the resulted permeance values are listed in TABLE II.

TABLE II: Leakage permeance values fitted to FEM $[W b / A]$

\begin{tabular}{c||c||c}
\hline$P_{s}$ & $P_{\text {long }}$ & $P_{\text {shunt }}$ \\
\hline \hline $100 \cdot 10^{-9}$ & $13.9 \cdot 10^{-9}$ & $30 \cdot 10^{-9}$ \\
\hline
\end{tabular}

For verification, other schemes are simulated with the leakage permeance values obtained from parameter fitting, and compared to the FEM result. The short-circuit inductances from FEM and PLECS circuit simulation are illustrated in Fig. 14 and Fig. 15. On one hand, Fig. 14 accounts for the scheme with the primary winding $W_{p}$ excited and single secondary winding $W_{s n}(n=1 \ldots 9)$ shorted. Except for the schemes that are used for parameter fitting of the leakage permeances (fit point 1 and 2), the short-circuit inductance values of the other test point matches quite well between FEM and PLECS. This U-shape curve reveals the fact that the more centralised the secondary winding is located with respect to the primary winding, the stronger is the coupling and thus the lower the short-circuit impedance will be. On the other hand, Fig. 15 accounts for the case when the secondary winding $W_{s 1}$ at the very top is excited, while the other 8 secondary windings are shorted one by one. Also in this case FEM and PLECS shows good match, and the rising curve demonstrates that the closer the secondary winding are located to each other, the better the coupling and the lower the short-circuit inductance value will 


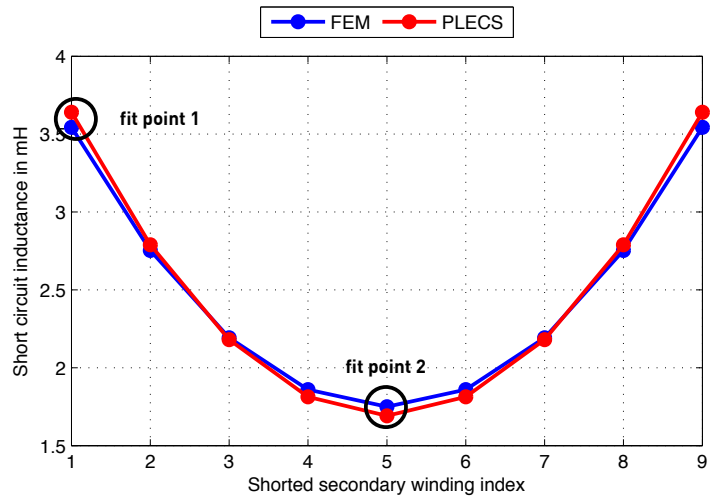

Fig. 14: Comparison to FEM with $W_{p}$ excited, $W_{s}$ shorted

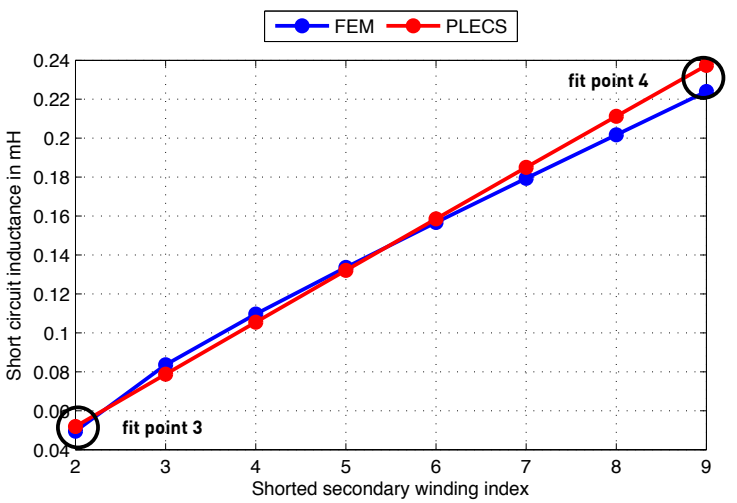

Fig. 15: Comparison to FEM, $W_{s 1}$ excited, other $W_{s}$ shorted

be. The simulation of all short-circuit combinations (e.g. $W_{s 2}$ excited and $W_{s 3}$ shorted) has resulted in a maximum error of 5\% between FEM and PLECS.

As a further step, the transformer prototype was tested experimentally as shown in Fig. 18. The bode analyser BODE 100 from Omicron has been used to measure the short circuit impedance at $50 \mathrm{~Hz}$, the inductive part of the impedance values was extracted for the parameter fitting and verification. The transformer prototype has undertaken the same short-circuit schemes as by FEM simulation. According to the four test points, the permeance magnetic circuit model has been fitted again as shown in Fig. 16 and Fig. 17. The leakage permeance values are listed in TABLE III

TABLE III: Leakage permeance values fitted to test $[W b / A]$

\begin{tabular}{c||c||c}
\hline$P_{s}$ & $P_{\text {long }}$ & $P_{\text {shunt }}$ \\
\hline \hline $3000 \cdot 10^{-9}$ & $440 \cdot 10^{-9}$ & $50 \cdot 10^{-9}$ \\
\hline
\end{tabular}

Attention needs to be paid that the short circuit inductance values from hardware test are significantly larger than that from FEM simulation. This is due to the fact that part of the windings exposes outside the window area of the core, which

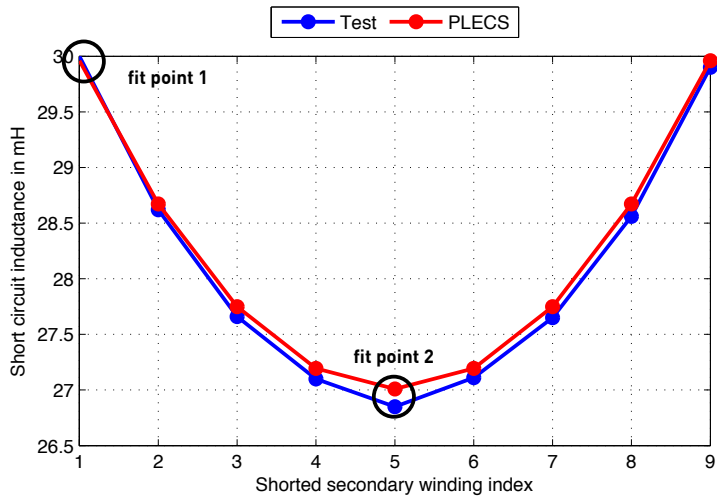

Fig. 16: Comparison to test with $W_{p}$ excited, $W_{s}$ shorted

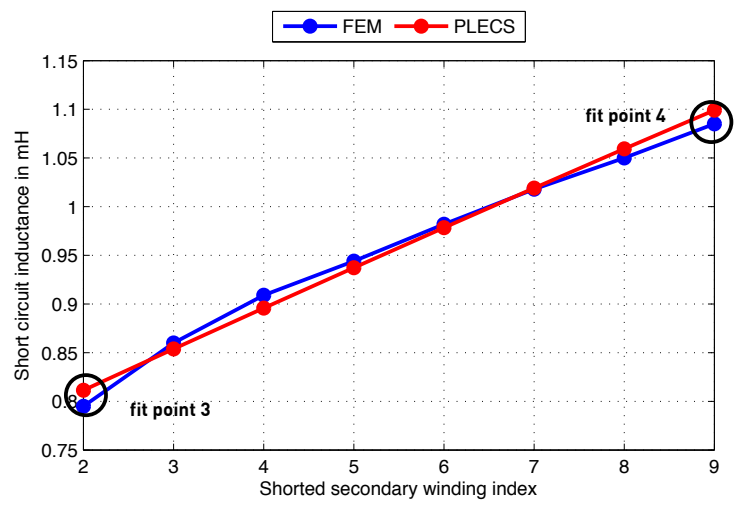

Fig. 17: Comparison to test, $W_{s 1}$ excited, other $W_{s}$ shorted

was not taken into account by the 2-D FEM model. This part of flux path can be modelled as an additional permeance network as depicted in Fig. 19, which is characterised by two groups of additional permeances $P_{s A n}$ and $P_{l o n g A n}$. Since $P_{s A n}$ and

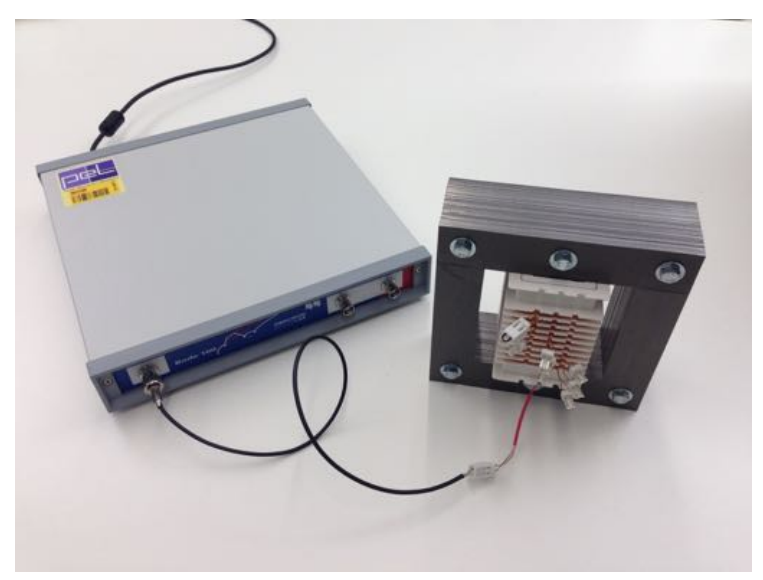

Fig. 18: Test environment of the transformer prototype 


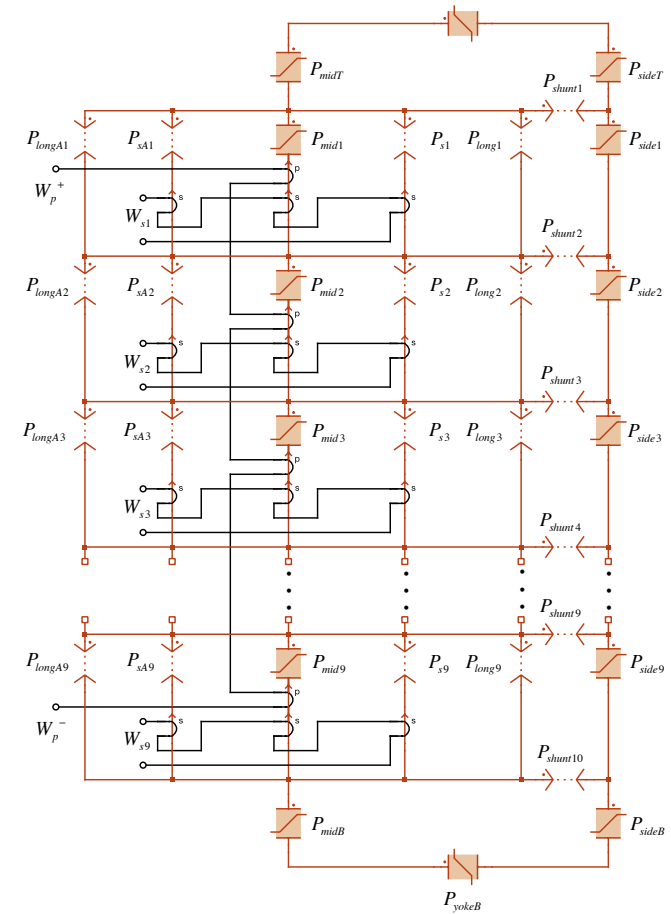

Fig. 19: Permeance circuit model of the prototype transformer including the air flux path outside the window area

$P_{\text {longAn }}$ are linear and can be merged in to $P_{s n}$ and $P_{\text {longn }}$, the final magnetic circuit should still have the same structure as in Fig. 13. In this way, the proposed model is still able to match the experimental test scheme quite well with an maximum error of $3 \%$

\section{CONCLUSion}

This paper has demonstrated the modelling of multiwinding transformer using permeance-based magnetic circuit, which can be seamlessly integrated into system-level simulation of power electronic, with direct interface to the electrical circuit. Via making use of information about the repetitive geometry, the parameter identification process of the leakage flux path can be much simplified. The result from the proposed model shows good match to both FEM simulation and hardware measurements, under two kinds of short-circuit test schemes. Future work will be invested in extending this approach to 3-phase case with different winding configurations (e.g. star, delta, mixed delta), and connection of power electronics converters on the secondary winding will be also a next step to further verify the fidelity.

\section{REFERENCES}

[1] C. Marxgut, J. Muehlethaler, F. Krismer, and J. W. Kolar, "Multiobjective optimization of ultraflat magnetic components with pcb-integrated core," in IEEE Transactions on Power Electronics, vol. 28, no. 7, 2013 , pp. 3591-3602.

[2] I. Hernandez, F. de Leon, J. Canedo, and J. C. Olivares-Galvan, "Modelling transformer cores joints using gaussian models for the magnetic flux density and permeability," in IET Electric Power Applications, vol. 4, 2010, pp. 761-771.

[3] J. van Vlerken and P. G. Blanken, "Lumped modelling of rotary transformers, heads and electronics for helical-scan recording," in IEEE Transactions on Magnetics, vol. 31, no. 2, 1995, pp. 1050-1055.

[4] J. Cale, S. D. Sudhoff, and L.-Q. Tan, "Accurately modeling ei core inductor using a high-fidelity magnetic equivalent circuit approach," in IEEE Transactions on Magnetics, vol. 42, no. 1, 2006, pp. 40-46.

[5] C. Alverez-Marino, F. de Leon, and X. M. Lopez-Fernandez, "Equivalent circuit for the leakage inductance of multiwinding transformers: unification of terminal and duality models," in IEEE Transactions on Power Delivery, vol. 27, no. 1, 2012, pp. 353-361.

[6] M. Lambert, M. Matinez-Duro, J. Mahseredjian, F. de Leon, and F. Sirois, "Transformer leakage flux models for electromagnetic transients: Critical review and validation of a new model," in IEEE Transactions on Power Delivery, vol. 29, no. 5, 2014, pp. 2180-2188.

[7] M. D. Manjrekar, P. K. Steimer, and T. A. Lipo, "Hybrid multilevel power conversion system: A competitive solution for high-power applications," in IEEE Transactions on Industry Applications, vol. 36, no. 3, 2011, pp. 834-841.

[8] M. Lambert, F. Sirois, M. Matinez-Duro, and J. Mahseredjian, “Analytical calculation of leakage inductance for low-frequency transformer modelling," in IEEE Transactions on Power Delivery, vol. 28, no. 1, 2013, pp. 507-515.

[9] D. Hamill, "Lumped equivalent circuits of magnetic components: the gyrator-capacitor approach," in IEEE Transactions on Power Electronics, vol. 8, 1994, pp. 97-103.

[10] J. Allmeling, W. Hammer, and J. Schönberger, "Transient simulation of magnetic circuits using the permeance-capacitance analogy," in Control and Modeling for Power Electronics (COMPEL), IEEE 13th Workshop on, 2012.

[11] E. C. Snelling, Soft Ferrite: Properties and Applications. London Iliffe Books Ltd., 1969.

[12] M. Luo and D. Dujic, "Permeance based modelling of the core corners considering magnetic material nonlinearity," in Annual Conference of the IEEE Industrial Electronics Society (IECON), 2015, pp. 950-955.

[13] B. Azzerboni, E. Cardelli, G. Finocchio, and F. L. Foresta, "Remarks about preisach function approximation using lorentzian function and its identification for nonoriented steels," in IEEE Transactions on Magnetics, vol. 39, no. 5, 2003, pp. 3028-3030. 